\title{
Stage IVA Thyroid Gland Anaplastic Carcinoma AJCC v7
}

National Cancer Institute

\section{Source}

National Cancer Institute. Stage IVA Thyroid Gland Anaplastic Carcinoma A/CC v7. NCI

Thesaurus. Code C87552.

Stage IVA includes: T4a, Any N, M0. T4a: Intrathyroidal anaplastic carcinoma. M0: No distant metastasis. (AJCC 7th ed.) 